\title{
Allergische Kontakturtikaria bei einem Reptilienhalter
}

\author{
Allergic Contact Urticaria in a Reptile Owner
}

Autoren

Institut

\section{B. Bleßmann-Gurk, B. Hoffmann, C. Bayerl}

Klinik für Dermatologie und Allergologie, Städtische Kliniken Wiesbaden HSK, Wilhelm-Fresenius-Klinik GmbH

\section{Bibliografie}

DOI $10.1055 / \mathrm{s}-2007-966354$

Akt Dermatol 2007; 33:

166-167 @ Georg Thieme

Verlag KG Stuttgart · New York ISSN 0340-2541

Korrespondenzadresse Dr. Birgit Bleßmann-Gurk Klinik für Dermatologie und Allergologie

Städtische Kliniken Wiesbaden Lehrkrankenhaus der

Universität Mainz

HSK, Wilhelm-Fresenius-Klinik

$\mathrm{GmbH}$

Aukammallee 39

65191 Wiesbaden

birgit.BLESSMANN-GURK@

HSK-wiesbaden.de

\section{Zusammenfassung \\ $\nabla$}

Die Kasuistik beschreibt eine Kontakturtikaria durch als Futtertiere verwendete Insekten bei einem Reptilienhalter. Im Nativ-Scratchtest wurden Sensibilisierungen gegen Mehlwürmer im Larvenstadium (Tenebrio molitor), Getreideschimmelkäfer im Larvenstadium (Alphitobius

\section{Einleitung \\ $\nabla$}

Die Freizeitgestaltung hat in unserer Gesellschaft einen großen Stellenwert. So rücken die Sensibilisierungen und klinisch relevanten Allergien erworben durch Hobbies zusehends in das allergologische Spektrum. Wir berichten über einen Reptilienzüchter mit Futtertier-assoziierten Sensibilisierungen, die zu einer akuten Kontakturtikaria führten.

\section{Anamnese}

$\nabla$

Ein 62-jähriger Reptilienhalter stellte sich mit immer wieder kurz nach der Fütterung seiner Reptilien (Schlangen etc.) aufgetretener Urtikaria vor. Die Symptomatik trat vor allem an Händen und Unterarmen, also den Kontaktstellen mit den Futtertieren, auf. Als Futtertiere wurden Mehlwürmer im Larvenstadium (Tenebrio molitor), Getreideschimmelkäfer im Larvenstadium (Alphitobius diaperinus), Schwarzkäfer im Larvenstadium (Zophobas morio) und Steppengrillen als adulte Tiere (Gryllus assimilis) sowie Mäuse und Ratten verwendet. Sensibilisierungen waren bereits gegen Dermatophagoides farinae (RAST-Klasse 4) und Dermatophagoides pteronyssinus (RAST-Klasse 3 ) nachgewiesen worden. Gegen Hundeepithelien waren Antikörper (RASTKlasse 1) ohne klinische Relevanz nachweisbar. Weiter bestand eine bekannte Rhinokonjunkti- diaperinus), Schwarzkäfer im Larvenstadium (Zophobas morio) und Steppengrillen als adulte Tiere (Gryllus assimilis) nachgewiesen. In der Literatur finden sich Beschreibungen von zumeist aerogenen Allergien gegen Insektenbestandteile. Eine Allergie gegen eine Grillenart wurde bisher nicht beschrieben.

vits allergica mit bekannten Sensibilisierungen gegen Frühblüher und Gräser. Nebenbefundlich fanden sich Insektengiftallergien (Biene und Wespe). Nach Hymenotperenstichen war es bisher nur zu Lokalreaktionen gekommen.

\section{Allergologische Testergebnisse $\nabla$}

Prick- und Reibetests wurden mit patienteneigenen Allergenen sowie kommerziellen Pricktestlösungen der Firmen Allergopharma (Reinbek) und ALK Scherax (Hamburg) durchgeführt. Als Kontrolle dienten $\mathrm{NaCl}$ 0,9\% sowie Histaminhydrochlorid $10 \mathrm{mg} / \mathrm{ml}$.

Scratchtestung mit Maus- und Rattenfell erbrachte keine positive Reaktionen. Pricktestung mit kommerziellen Extrakten aus Dermatophagoides pteronyssinus, Dermatophagoides farinae, Tyrophagus putrescentiae und Acarus siro zeigte jeweils 2-fach positive Reaktion.

Scratchtestung nativ mit einer Steppengrille (Gryllus assimilis), einem Schwarzkäfer im Larvenstadium (Zophobas morio), einem Mehlwurm im Larvenstadium (Tenebrio molitor) sowie einem Getreideschimmelkäfer im Larvenstadium (Alphitobius diaperinus) führten zu ausgeprägten, 3-fach positiven Reaktionen, teils mit Pseudopodien ( $\bullet$ Abb. 1 und $\odot$ 2). Bei einer Kontrollperson, ebenfalls mit nachgewiesene Allergie gegen Dermatophagoides spezies, ließen sich im Scratchtest keine positiven Reaktionen gegen 


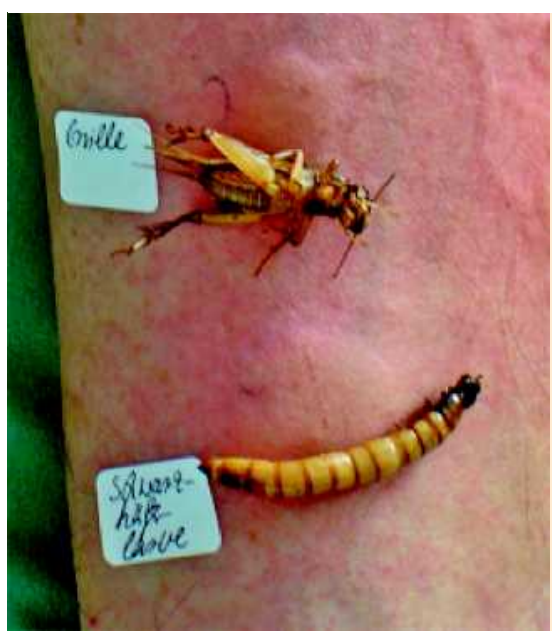

Abb. 1 Nativer Scratchtest mit einer Steppengrille und einer Schwarzkäferlarve mit ausgeprägter urtikarieller Reaktion und Pseudopodien.

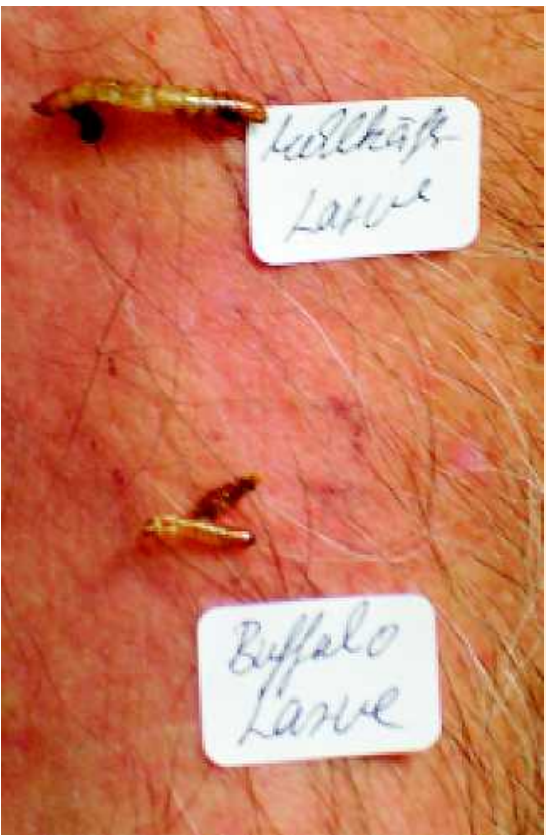

Abb. 2 Nativer Scratchtest mit einer Mehlkäferlarve und einer Getreideschimmelkäferlarve $=$ Buffalo Larve.

Gryllus assimilis, Zophobas morio, Tenebrio molitor und Alphitobius diaperinus nachweisen.

\section{Laborchemische Ergebnisse \\ $\nabla$}

Spezifisches IgE (CAP) (Bioscentia Ingelheim) gegen Rattenepithelien, Rattenepithelien und Serumprotein, Mäuseepithelien und Serumprotein nicht nachweisbar. Gegen Wüstenspringmaus war CAP-Klasse $1(0,41 \mathrm{kU} / \mathrm{l})$ nachweisbar.

\section{Diskussion}

$\nabla$

Die allergologische Diagnostik bei unserem Reptilienhalter zeigte eindeutige Sensibilisierungen gegen die Körperhüllenbestandteile der als Futtertiere verwendeten Insekten. In der Literatur wurden bereits Einzelfälle von Kontakturtikaria durch Tenebrio molitor beschrieben. In diesen Fällen war die Hauttestung mit Ganzkörperextrakten positiv verlaufen [1]. Aerogene und ingestive Sensibilisierungen gegen Bestandteile von Tenebrio molitor und Zophobas morio sowie andere Insekten sind beschrieben [2]. Häufig ist die gleichzeitige Sensibilisierung auf Milbenarten und Insektenbestandteile. Diese Berichte beziehen sich auf Arbeiter in der Landwirtschaft. In diesen Fällen standen jedoch respiratorische Symptome im Vordergrund [3]. Beschreibungen über Sensibilisierungen gegen eine Grillenart finden sich nach unseren Recherchen in der Literatur bisher nicht.

Wir empfahlen unserem Patienten, die Reptilien nur noch unter Verwendung von Einmalhandschuhen zu füttern und danach die Hände zu waschen. Der Patient gab ein Verschwinden der Beschwerden an. Aufgrund der Sensibilisierungen gegen Hausstaub-, Schmutz- und Speichermilben besprachen wir Maßnahmen zur Allergenkarenz. Berichte zu Kreuzallergien zwischen Insektenbestandteilen und Milbenarten fanden sich in der Literatur nicht.

Die Kasuistik zeigt, wie wichtig die allergologische Diagnostik mit patienteneigenen Materialien ist, insbesondere bei speziellen Fragestellungen. Irritative und somit falsch positive Reaktionen sind durch Hinzuziehen von Kontrollpersonen auszuschließen. Die Frage nach Hobbies ist Bestandteil einer umfassenden dermato-allergologischen Anamnese.

\section{Abstract}

\section{Allergic Contact Urticaria in a Reptile Owner $\nabla$}

This case report focuses on contact urticaria of a reptile owner after feeding his reptiles. Scratch tests with native materials revealed sensibilisation to larvae of mealworms (Tenebrio molitor), of darkling beetles (Alphitobius diaperinus), of superworms (Zophobas morio) and adult prairie cicadas (Gryllus assimilis). In literature, persons with aerogen sensibilisation to Tenebrio molitor and Zophobas morio are described. Allergy towards prairie cicadas has not been described hitherto.

\section{Literatur}

1 Bygum A, Bindslev-Jensen C. Mealworm allergy. Ugeskrift for Laeger 1997; 159: $6532-6533$

2 Freye $H B$, Esch RE, Litwin CM, Sorkin L. Anaphylaxis to the ingestion and inhalation of Tenebrio molitor (mealworm) and Zophobas morio (superworm). Allergy Asthma Proceedings 1996; 17: 215 - 219

3 Armentia A, Martinez A, Castrodeza R, Martinez J, Jimeno A, Mendez J, Stolle $R$. Occupational allergic disease in cereal workers by stored grain pests. Journal of Asthma 1997; 34: 369- 378 